\title{
LA REALIDAD DEL MERCADO INFORMAL DE QUITO EN TIEMPOS DE PANDEMIA COVID-19, 2020
}

\section{THE REALITY OF THE INFORMAL MARKET IN QUITO IN TIMES OF THE COVID-19 PANDEMIC, 2020}

\section{Mario Andrés Miranda Guatumillo ${ }^{1 \star}$, Bernardo Zambrano Velasco ${ }^{2}$, Esther Briggitte Castellanos Espinoza ${ }^{3}$, Katheryn Zatizabal Morán ${ }^{4}$}

\section{1,2,3,4 - Investigador independiente, Quito, Ecuador}

1. Email: andresmirandag97@gmail.com ORCID: https://orcid.org/0000-0002-8404-1795

2. Email: bernardo.zambranov@outlook.com ORCID: https://orcid.org/0000-0002-8430-3951

3. Email: ecastellanos1087@gmail.com ORCID: https://orcid.org/0000-0001-7181-5096

4. Email: katheryn_zm@hotmail.com ORCID: https://orcid.org/0000-0001-7657-5423

Recibido: 01/02/2021 Aceptado: 25/06/2021

Para Citar: Miranda Guatumillo, M. A., Zambrano Velasco, B., Castellanos Espinoza, E. B., \& Zatizabal Morán, K. (2021). La realidad del mercado informal de Quito en tiempos de pandemia COVID-19, 2020. Revista Publicando, 8(30), 47-56. https:// doi.org/10.51528/rp.vol8.id2187

\section{Resumen:}

El año 2020 fue un periodo complicado para la economía mundial debido a la emergencia sanitaria producida por la pandemia COVID-19, no siendo la excepción los países de América Latina ni Ecuador. En el caso de Quito, capital de Ecuador, la incidencia de la pandemia ha hecho que los niveles de desempleo aumenten, y por tanto, la informalidad también. La ciudad ya venía atravesando en los últimos años serios problemas en la política laboral donde su economía informal es la segunda más representativa a nivel nacional, después de Guayaquil. Por ello, es importante conocer cómo se ha visto afectado el mercado informal de Quito en el año 2020. Para la obtención de los datos, se aplicó un enfoque cualitativo y el diseño de investigación de carácter documental y bibliográfico, siendo las principales fuentes el Instituto Nacional de Estadísticas y Censo (INEC) y la Encuesta Nacional Empleo, Desempleo y Subempleo (ENEMDU). La investigación tiene como conclusión que el 13,90\% de la Población Económicamente Activa (PEA) de Quito pertenece al mercado informal, siendo en su mayoría jóvenes, quienes se dedican a vender productos como ropa, alimentos, entre otros, de forma ambulante. Así mismo, existen 9.606 comerciantes informales en la ciudad de Quito, de los cuales, el 70\% de los comerciantes informales cuentan con permisos para vender, mientras que el 30\% restante son autónomos y no están regulados.

Palabras clave: Pandemia, Mercado informal, Vendedor ambulante, COVID-19.

\begin{abstract}
:
The year 2020 was a complicated period for the world economy due to the health emergency produced by the COVID-19 pandemic, and Latin American countries and Ecuador were no exception. In the case of Quito, capital of Ecuador, the incidence of the pandemic has caused unemployment levels to increase, and therefore, informality as well. The city had already been going through serious problems in labor policy in recent years, where its informal economy is the second most representative at the national level, after Guayaquil. Therefore, it is important to know how Quito's informal market has been affected in 2020. In order to obtain the data, a qualitative approach was applied and the research design was documentary and bibliographic, being the main sources the National Institute of Statistics and Census (INEC) and the National Survey of Employment, Unemployment and Underemployment (ENEMDU). The research concludes that $13,90 \%$ of the Economically Active Population (EAP) of Quito belongs to the informal market, being mostly young people, who are engaged in selling products such as clothing, food, among others, on the street. Likewise, there are 9.606 informal traders in the city of Quito, of which 70\% of informal traders have permits to sell, while the remaining $30 \%$ are self-employed and are not regulated.
\end{abstract}

Keywords: Pandemic, Informal market, Peddler, COVID-19. 


\section{INTRODUCCIÓN}

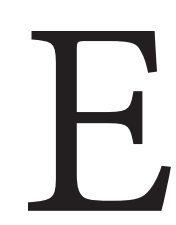
1 campo de estudio de la economía laboral es bastante amplio pues comprende desde el escogimiento de las personas respecto a sus labores, las necesidades de mano de obra de las distintas unidades de producción con sus respectivas especificaciones, los sueldos y salarios de los mismos, la participación de gremios y sindicatos y la presión que ejercen en el mercado de trabajo, planificación de fuentes de empleo, distribución de ingresos, mecanismos de contratación, condiciones y ambientes laborales, proporción de empleo, subempleo y desempleo.

Los fundamentos de la economía clásica se centran mucho en los economistas Adam Smith (1723-1790) y David Ricardo (1772-1823) cuya teoría central está basada en la libre economía y se la alcanza a través de las fuerzas del mercado dando lugar a que el trabajo genere una retribución llamada sueldo o salario, que se produce por el encuentro de la oferta y demanda alcanzando el punto de equilibrio. Smith considera que la riqueza de un país puede aumentar si el estado no interviene en la economía, por tanto el orden natural económico no se ve afectado. Según Smith (1980):

Los salarios del trabajo, en todas las ocasiones, se acomodan al convenio que por común se hace entre estas dos partes, cuyos intereses de ningún modo pueden considerarse los mismos. El operario desea sacar lo más y el empresario dar lo menos que pueda. Los primeros están siempre dispuestos a concertar medios de levantar y los segundos de bajar, los salarios del trabajo (p. 70).

David Ricardo estudia los sueldos y salarios en la Teoría de distribución. Según Ricardo (1973),
"El precio de mercado de la mano de obra es el precio que realmente se paga por ella, debido al juego natural de la proporción que existe entre la oferta y la demanda" (p. 71).

En América Latina se ha visto afectado el mercado laboral y sus relaciones desde la década de 1980 debido a la flexibilización el trabajo y su precarización, las relaciones laborales han sido afectadas por la desregulación y la protección al trabajador ha disminuido con la intención de minimizar los costos empresariales para que así las compañías puedan incrementar sus réditos y se incentive tanto la inversión local como extranjera. Con la llegada del neoliberalismo, el entorno laboral de la región se ha vuelto mayormente heterogéneo lo que produce que la calidad de vida se vea menoscabada. Las medidas de desregularizar, privatizar y que primen las reglas de mercado han sido consideradas necesarias para que se pueda dar el crecimiento en América Latina. Sin embargo, este desarrollo de la región se ve contrastado por la informalidad laboral y la precarización que se enfrenta, estos acontecimientos no son nuevos y muy por el contrario han ido en aumento.

El mercado de trabajo alcanza su equilibrio cuando la oferta y demanda laboral se interceptan dando lugar a una cantidad laboral y salario óptimo, pero la realidad es que este mercado no siempre alcanza esos niveles de equilibrio puesto que las personas que desean acceder al mercado laboral suelen exceder a las plazas existentes, lo que termina generando una economía informal. Según Villarán (2019), "La informalidad como concepto aparece en los años 70 del siglo pasado. El primero que utilizó éste término, fue el investigador Keith Hart para explicar el empleo 
urbano en las ciudades del África.", la Organización Internacional del Trabajo (OIT) promovió el concepto de "Sector Informal Urbano" en Kenia el cual fue utilizado en la academia.

El mercado informal está compuesto por personas o empresas que ejercen actividades de compra-venta cuyos bienes o servicios objeto de comercialización no se encuentran debidamente registrados y por tanto no existe el pago de impuestos por dicha actividad. En este mercado es difícil obtener datos respecto a los ingresos generados por lo que se trabaja mucho con datos estadísticos estimados. El sector informal es definido por Estupiñán y Pincay (2020) “El conjunto de actividades económicas no reguladas por el Estado que realizan pequeñas empresas y trabajadores autónomos de bajos recursos para generar ingresos y cubrir sus necesidades sin contar con la protección y los beneficios que ofrece la ley" (p. 29).

La economía ecuatoriana y su mercado laboral sufrieron un revés sin precedente en el año 2020 debido a la emergencia sanitaria de la COVID-19. Según proyecciones de CEPAL, Comisión Económica para América Latina y el Caribe (2020) Ecuador sufriría una contracción del 9.5\% lo que ocasiona que la tasa de desempleo se incremente en al menos 3.4\% hasta llegar al límite del 11.5\% lo cual genera menos empleo y menos horas de trabajo, además de lo mencionado se espera que la calidad de empleo se vea mermada.

La informalidad laboral genera fuentes de ingreso, los cuales en su mayoría son bajos y volátiles, y cada vez se vuelve más común en Ecuador debido a la incertidumbre por las pocas plazas de trabajo ofertadas en los últimos tiempos las cuales en la actualidad se han visto más contraídas por la pandemia existente de COVID19. El aumento de la informalidad en la nación también se da por la llegada de extranjeros quienes no cuentan con la documentación requerida para aspirar a un empleo formal y aun así se ven en la necesidad de trabajar informalmente para subsistir. Las personas que deciden ingresar a la economía informal lo hacen por necesidad de sobrevivencia por tanto los ingresos generados son limitados e inestables lo que no permite sobrellevar periodos prolongados de inactividad, tampoco se cuenta con el acceso a seguridad y salud. Alberto Acosta (como se citó en Alvarado, 2020), director de la publicación económica Análisis Semanal, explicó que el "aumento del "otro empleo no pleno" se explica porque hubo personas que perdieron su empleo formal y trataron de generar ingresos para sobrevivir o que dejaron de buscar un empleo en mejores condiciones y se conformaron con un trabajo precario".

El país así como el resto del mundo se vio afectado por el confinamiento debido a la pandemia que aún persiste, esto no fue impedimento para que los vendedores informales siguieran trabajando, infringiendo muchas veces con las medidas de bioseguridad y el desacatamiento de las órdenes gubernamentales en la prevención y detención de más contagios por COVID-19. En el presente artículo se analizará el efecto de la pandemia en el mercado informal de la ciudad de Quito, capital de Ecuador.

\section{MATERIALES Y MÉTODOS}

El trabajo se realizará con un enfoque 
cualitativo y el diseño de la investigación será documental y bibliográfico, puesto que se realizó un estudio hermenéutico de las teorías de economía clásica y sobre el mercado informal. La recolección de datos es mediante la documentación y según Hernández, Fernández y Baptista (2014), "prácticamente la mayoría de las personas, grupos, organizaciones, comunidades y sociedades los producen y narran, o delinean sus historias y estatus actuales" (p. 415). Se considera como fuente de investigación los datos de la encuesta Nacional de Empleo, Desempleo y Subempleo (ENEMDU) del Instituto Nacional de Estadísticas y Censos (INEC), así como también se utilizará la observación como fuente de recolección complementaria pues permitirá evidenciar la realidad de los vendedores informales de la ciudad de Quito.

\section{RESULTADOS}

El mercado informal está compuesto por personas que no tienen empleo y por tanto no cuentan con un sueldo o salario establecido, quienes deciden ejercer actividades de venta de bienes y/o servicios que no poseen regulación o respaldo por parte del estado, conciben este tipo de actividad como una oportunidad para generar recursos y así poder solventar sus gastos diarios. Estas personas que forman parte del mercado informal generalmente son consideradas como vendedores ambulantes. Según Bromley (2000):

Los vendedores ambulantes son un componente integral de las economías urbanas alrededor del mundo. Como distribuidores de mercancías y servicios asequibles, estos trabajadores proporcionan a los consumidores opciones convenientes y accesibles de comercio minorista y forman una parte vital de la vida económica y social de una ciudad. La venta ambulante como ocupación ha existido por cientos de años (pp. 128).

Previo a la crisis económica ocasionada por el coronavirus, Ecuador atravesaba un estancamiento serio en la economía y la tasa de empleo informal era alta. Para septiembre 2020, el empleo adecuado representaba el $32.1 \%$ de la Población Económicamente Activa (PEA) como se visualiza en la Figura 1.

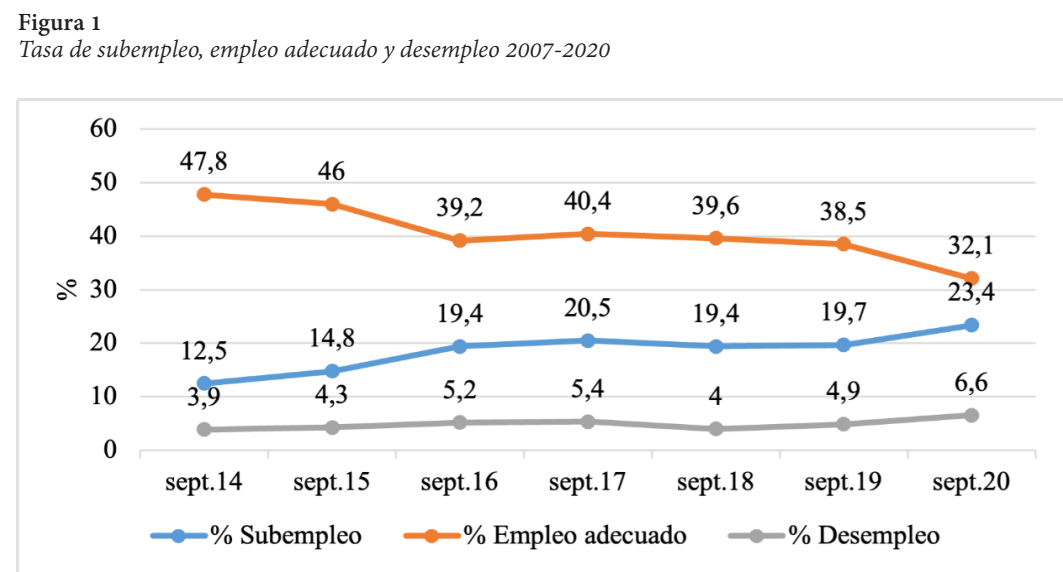

Nota: Datos tomados de la Encuesta Nacional de Empleo, Desempleo y Subempleo (ENEMDU) del Instituto Nacional de Estadísticas y Censos (INEC). 
En la Figura 2 se presenta la tasa de subempleo para las principales ciudades del Ecuador del año 2020.

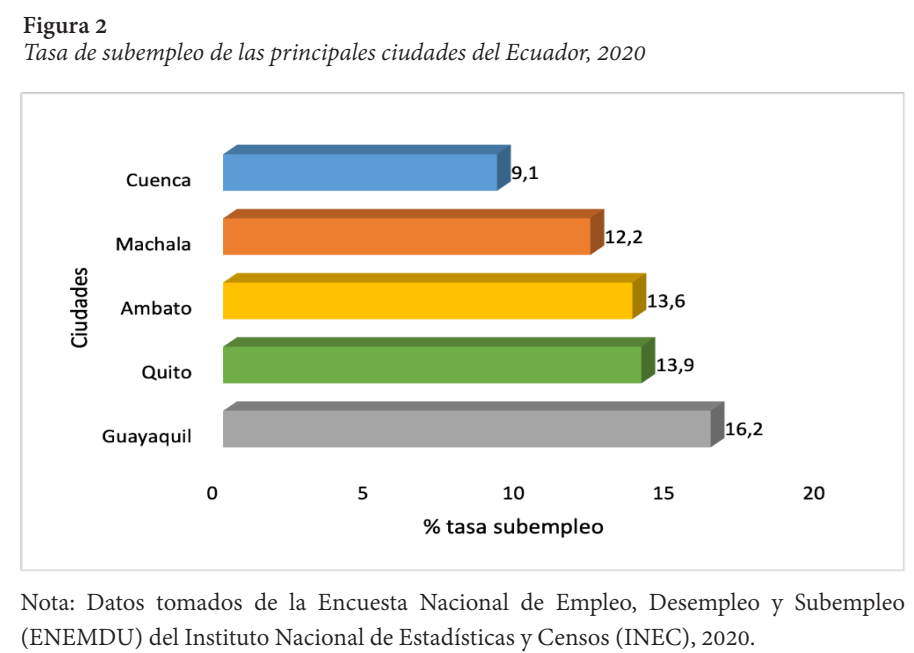

En el gráfico anterior se observa que en la ciudad de Quito, el $13.90 \%$ de la PEA pertenece al mercado informal; es decir, se dedican a la venta de ropa, calzado, alimentos, artículos de segunda mano, cigarrillos y demás artículos varios que no son regulados. La Ordenanza Metropolitana 280 (ORDM-0280, 2012) considera el trabajo autónomo en la ciudad de Quito y clasifica a los comercios informales de la siguiente manera:

1. Trabajadores autónomos fijos - Son aquellos que se encuentran en espacios de uso público fijos, ubicados en una jurisdicción administrativa zonal delimitada con calle principal y secundaria. Se considerará también un sistema rotativo, de conformidad con la normativa de ejecución respectiva.

2. Trabajadores autónomos semifijos - Son aquellos que laboran en una jurisdicción administrativa zonal, en un radio de acción determinado y por un tiempo establecido.

3. Trabajadores autónomos ambulantes Son aquellos que se desplazan por todo el territorio del Distrito Metropolitano de Quito.

4. Trabajadores autónomos ocasionales y temporales - Son aquellos que laboran en sitios específicos, así como en ferias y espectáculos públicos durante la realización de eventos, por ocasión y temporadas.

5. Trabajadores autónomos en transportación pública - Son aquellos que realizan sus actividades de comercio en el interior de las unidades de transportación pública en el Distrito Metropolitano de Quito.

Según la Agencia Metropolitana de Control (AMC, 2020) existen 9,606 comerciantes informales en la ciudad de Quito, de los cuales el $70 \%$ si tienen los permisos para trabajar mientras que el resto son comerciantes autónomos no regulados. Quito, a nivel de empleo, depende mucho de lo que suceda en el sector público ya que concentra la mayor cantidad de instituciones del Gobierno central por tanto si este sector se ve afectado inmediatamente se refleja un impacto en la dinámica laboral de la ciudad la cual ya venía 
teniendo reveses desde el año 2016 por los diferentes recortes laborales que han existido, lo sucedido con la pandemia hizo que estos indicadores se agudizaran, ya que el sector laboral de la ciudad no se centra en producción ni exportación. No obstante, el problema de informalidad de Quito está latente desde antes de la pandemia, no existe información exacta de la localización de los comerciantes pero por observación refleja mayor concentración en los sectores de: Calderón, Cotocollao, Naciones
Unidas, La Carolina. El Ejido, Plaza Grande, Chillogallo, El Recreo, Mercado Mayorista, El Camal.

En la Figura 3 se muestra la composición del comercio informal de la ciudad de Quito, donde se ve reflejado que el $55 \%$ de los mismos corresponden a vendedores ambulantes, cabe señalar que la edad promedio de los comerciantes informales de la ciudad de Quito es de 35 años, mientras que la proporción de hombres y mujeres es equilibrada.

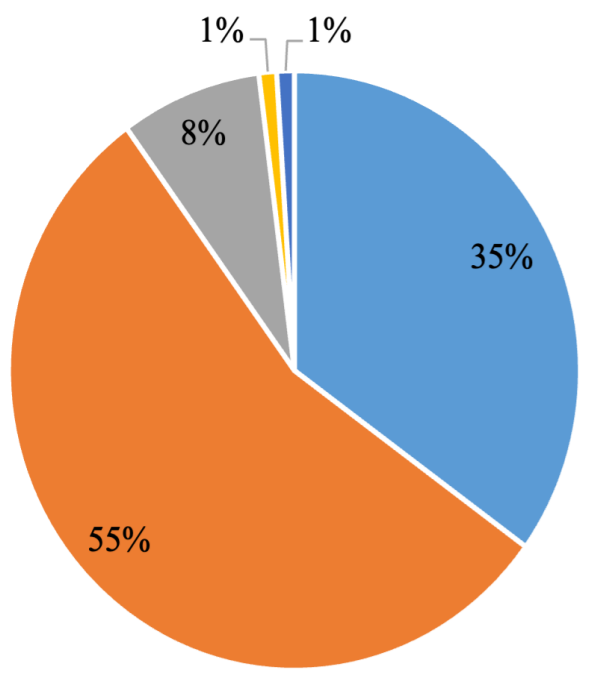

- Trabajador autónomo fijo

- Trabajador autónomo ambulante por ciertos sectores de la ciudad

- Trabajador autónomo ambulante por toda la ciudad

- Trabajador autónomo ocasional en ferias o espectáculos

- Trabajador autónomo que labora en el transporte público

Nota: Datos tomados del informe “Una mirada al empleo informal en Quito” por Grupo Faro, 2020.

La inmigración también ha afectado el mercado laboral ecuatoriano, no siendo la excepción el sector de comerciantes informales de Quito, donde los vendedores de nacionalidad ecuatoriana representan aproximadamente las dos terceras partes de los comerciantes autónomos y el tercio restante son de nacionalidad extranjera predominando la nacionalidad venezolana, también existen inmigrantes colombianos, peruanos, cubanos, entre otros, como se muestra en la Figura 4. 


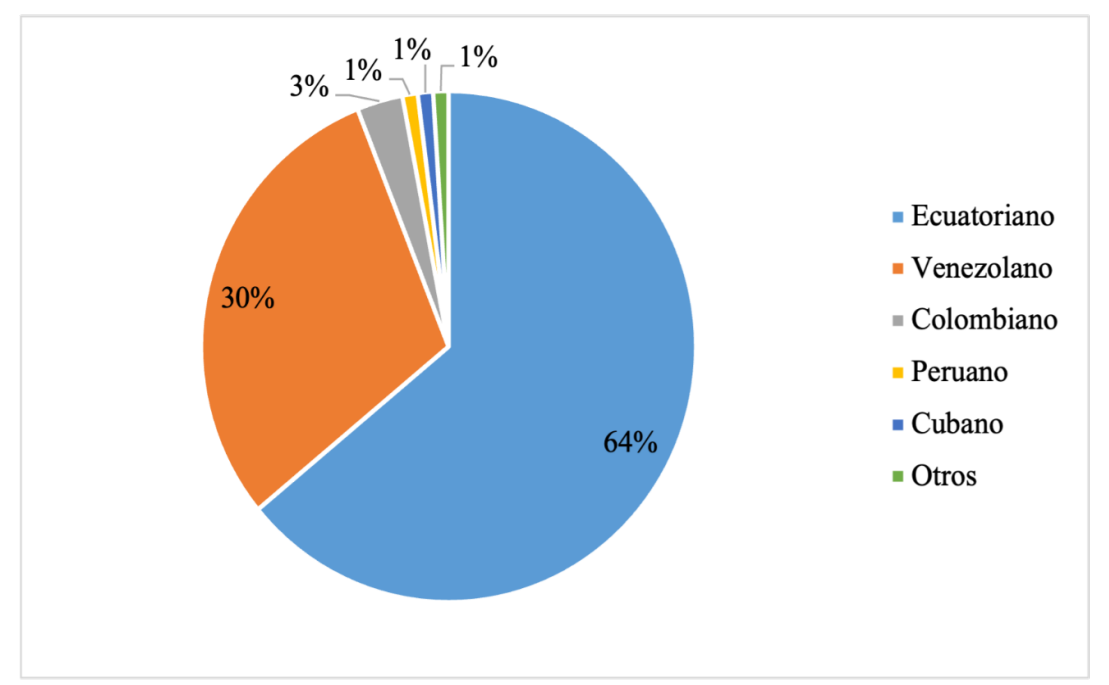

Nota: Datos tomados del informe "Una mirada al empleo informal en Quito" por Grupo Faro, 2020.

Según datos de INEC (2020) el ingreso mensual promedio antes de la emergencia sanitaria era de $\$ 302$, el cual disminuyó aproximadamente en un $46.36 \%$ obteniendo en promedio después de la emergencia un ingreso de $\$ 162$. Hay que recalcar que este ingreso percibido es inferior al salario básico unificado y a la canasta básica, no obstante el gobierno ofrece su ayuda a través del Bono de Desarrollo Humano y el Bono que otorga el gobierno por la emergencia sanitaria. No obstante es evidente la contracción de los ingresos percibidos por los comerciantes informales durante la pandemia COVID-19 a pesar que sus horas de trabajo se han incrementado para tratar de subsistir el duro momento de la emergencia. Este aumento en horas de trabajo a la semana no evidencia incremento en los ingresos, muy por el contrario estos han descendido, aproximadamente el $80 \%$ de los comerciantes informales le dedican al menos 6 días a la semana al desarrollo de la actividad. En la siguiente tabla resumen se muestran los ingresos, gastos, ganancia neta y horas de trabajo en un comparativo antes y desde la pandemia.

Tabla 1

Comparativo ingresos diarios antes y durante la pandemia

\begin{tabular}{|c|c|c|c|c|}
\hline \multirow{2}{*}{$\begin{array}{l}\text { Ingreso diario } \\
\text { Día alto }\end{array}$} & \multicolumn{2}{|c|}{ Antes de pandemia } & \multicolumn{2}{|c|}{ Después de pandemia } \\
\hline & $\$$ & 20.00 & $\$$ & 12.00 \\
\hline Día bajo & $\$$ & 10.00 & $\$$ & 5.00 \\
\hline Día normal & $\$$ & 15.00 & $\$$ & 8.00 \\
\hline Horas de trabajo a la semana & \multicolumn{2}{|c|}{47} & \multicolumn{2}{|c|}{51} \\
\hline Gasto diario & $\$$ & 5.00 & $\$$ & 3.00 \\
\hline Ganancia diaria & $\$$ & 10.00 & $\$$ & 5.00 \\
\hline
\end{tabular}

Nota. Datos tomados del informe “Una mirada al empleo informal en Quito" por Grupo Faro, 2020. 
El Municipio de Quito da a los vendedores ambulantes (comerciantes que están en las calles, transporte y espacio público) un Permiso Único de Comercio Autónomo (PUCA), este permiso va dirigido a las personas que van a tener un espacio determinado para su actividad comercial. También hay los permisos móviles pero estos son muy restringidos.

Aproximadamente los dos tercios de los comerciantes no poseen permiso, mientras que el $22 \%$ de los mismos estuvo interesado en obtenerlo, no obstante no se lo dieron. Esto se aprecia en la
Figura 5. Los vendedores desconocen de los procesos para la obtención de permisos, algunos si los conocen pero manifiestan no cumplir con los requisitos y otros consideran que no es necesario para sus actividades ambulantes y esta situación se ve agravada pues no cuentan con los fondos necesarios para el pago de los trámites de gestión para la obtención de los permisos. Solo el $11 \%$ de los trabajadores informales cuentan con permiso para laborar de los cuales el $73 \%$ de este segmento poseen permisos permanentes mientras que el $27 \%$ es temporal.

Figura 5

Permisos de funcionamiento

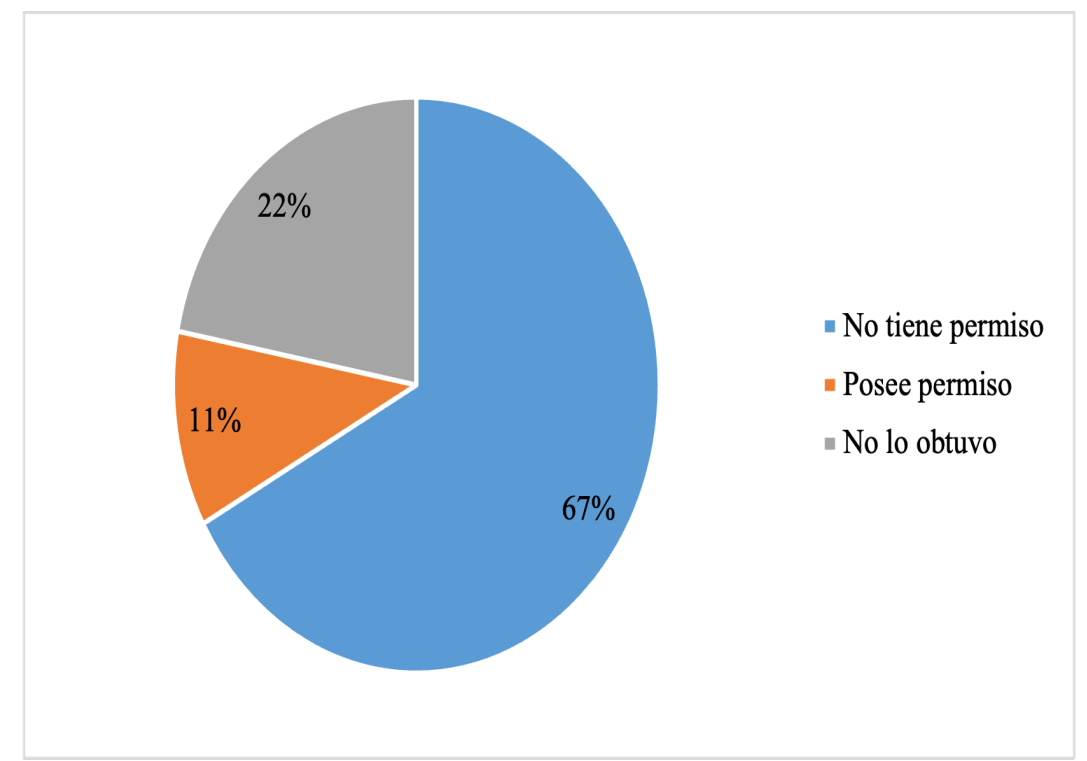

Nota: Datos tomados del informe “Una mirada al empleo informal en Quito" por Grupo Faro, 2020.

\section{CONCLUSIONES}

El mercado informal es parte de la Población Económicamente Activa (PEA) y la ciudad de Quito posee la segunda tasa más alta de subempleo en el país, ubicándose después de Guayaquil con un $13.90 \%$. Ecuador enfrenta problemas en su mercado laboral puesto que el empleo adecuado ha ido disminuyendo en los últimos años y esta falencia se ha visto magnificada por la emergencia sanitaria que se atraviesa actualmente por la pandemia del coronavirus. Quito, al ser la capital del país, concentra el mayor número de instituciones públicas lo que hace que su mercado 
de trabajo se concentre en este sector el cual ha sido afectado durante el último gobierno por los recortes de personal obligando a acrecentar más la brecha entre el empleo adecuado y el mercado informal el cual está compuesto en su mayoría por jóvenes quienes se han visto obligados a laborar más horas por una menor cantidad de ingreso mensual, es drástico el decrecimiento de los ingresos durante la pandemia sin considerar que los mismos no son equivalentes ni siquiera al salario básico unificado.

También es considerable la participación de población extranjera en el mercado informal de Quito, aproximadamente un tercio de los comerciantes ambulantes son extranjeros y al no existir una regularización gubernamental efectiva hace que exista precarización laboral. Es evidente que a pesar que el Municipio de Quito otorga permisos de funcionamiento, la mayoría de comerciantes no acceden a ellos ya sea por desconocimiento o por falta de recursos.

La COVID-19 sólo vino a evidenciar exponencialmente las debilidades que ya existían desde antes de la emergencia sanitaria, ni siquiera el otorgamiento de los distintos bonos gubernamentales logra disminuir la disparidad existente en el mercado informal de Quito, que lamentablemente no solo afecta a esta ciudad sino que la capital es el reflejo de lo que actualmente está aconteciendo en el país, por tanto es inminente que el Gobierno genere medidas para mejorar esta situación que ya se viene acarreando en el último quinquenio y así lograr que ese creciente mercado informal disminuya. 


\section{$\begin{array}{lllllll}\text { R } & \text { E } & \text { V } & \text { I } & S & \text { T } & \text { A }\end{array}$}

Public a n d o

I S S N $13390-9304$

\section{REFERENCIAS BIBLIOGRÁFICAS}

Agencia Metropolitana de Control (AMC, 2020). Informe de calidad de vida 2020. https:// quitocomovamos.org/wp-content/ uploads/2020/12/INFORME-DECALIDAD-DE-VIDA-QUITO-COMOVAMOS_compressed-3.pdf

Alvarado, P. (2020, enero 21). El empleo formal se mantuvo en Guayaquil. El Comercio. https://www.elcomercio.com/ actualidad / e mpleos - guayaquil economia-ecuador-formalidad.html

Bromley, R. (2000). Women in International Employment: Globalizing and Organizing Street Vending and Public Policy: A Global Review. Journal of Sociology and Social Policy, 20(1/2), 1-28

Comisión Económica para América Latina y el Caribe CEPAL (2020). Balance Preliminar de las Economías de América Latina y el Caribe 2020. https:// repositorio.cepal.org/bitstream/handle/ $\begin{array}{lllllllllllllll}1 & 1 & 3 & 6 & 2 & / & 4 & 6 & 5 & 0 & 1 & / & 1 & 4\end{array} /$ BP2020_Ecuador_es.pdf

Estupiñán, M., y Pincay, C. (2020). Marketing Social aplicado al mercado informal de la ciudad de Guayaquil. Universidad de Guayaquil.

Grupo Faro (2020). Una mirada al empleo informal en Quito. https:// grupofaro.org/wp-content/uploads/ $2020 / 12 /$ Una-mirada-al-empleoinformal-en-Quito_compressed-1.pdf
Hernández, R., Fernández, C., y Baptista, P. (2014). Metodología de la Investigación (6 ed.). México D.F., México: Editorial, McGrawHill Education.

Instituto Nacional de Estadísticas y Censos INEC (2020). ENEMDU, Encuesta Nacional de Empleo, Desempleo y Subempleo. https:// www.ecuadorencifras.gob.ec/empleoseptiembre-2020/

Ordenanza Metropolitana 0280. (2012, 7 de septiembre). Comercio Quito. Art. 4 http://www.comercio.quito.gob.ec/ i mage s / bas elegal/ O R D M - 0280 DESARROLLO-INTEGRAL-DE-LOSTRABAJADORES-AUTONOMOS.pdf

Ricardo, D. (1973). Principios de economía política y tributación. México: Fondo de Cultura Económica.

Smith, A. (1980). Riqueza de las naciones. México: Cruz O.S.A.

Villarán, F. (2019, mayo 13). Las causas de la informalidad. RPP Noticias. https:// $\mathrm{r} \mathrm{p} \mathrm{p} \cdot \mathrm{p}$ e / c o l u m n i s t a s / fernandogonzalovillarandelapuente/lascausas-de-la-informalidad-noticia1195869 